\title{
ADJOINT-BASED CONTROL OF AFTERBODY FLOW UNSTEADINESS IN THE COMPRESSIBLE REGIME
}

\author{
Ph. Meliga ${ }^{1}$, D. Sipp ${ }^{2}$, and J.-M. Chomaz ${ }^{1}$ \\ ${ }^{1}$ LadHyX-CNRS, Ecole Polytechnique \\ Palaiseau, France \\ ${ }^{2}$ ONERA \\ Département d'Aérodynamique Fondamentale et Expérimentale \\ Meudon, France
}

The theoretical study of a compressible afterbody flow in the subsonic regime is presented. It relies on sensitivity analyses developed in the framework of the linear global stability theory and aims at predicting beforehand the effect of a steady bulk and wall forcing on the growth rate of linear global modes. Such an analysis stands as a step in the perspective of a full control of afterbody flow unsteadiness. The sensitivity functions are derived analytically using adjoint methods and presented for the global mode responsible for the onset of a periodic regime. Various control methods are investigated, including an additional control device in the lee of the main body, heat sources, and boundary forcing. The obtained results show that the global mode is sensitive to momentum forcing along the separation line, to a localized heating in the core of the recirculating bubble, and to a blowing velocity close to the edge of the base.

\section{INTRODUCTION}

Open-loop control relies on a simple idea that a fixed modification in the flow conditions is susceptible to affect the whole flow dynamics. In the context of the cylinder wake flow, Strykowski and Sreenivasan [1] have experimentally investigated how a small control cylinder could alter the vortex-shedding phenomenon if suitably placed in the lee of the main cylinder. These authors successfully identified flow regions where the addition of the control cylinder leads to a complete suppression of the phenomenon. 
Such an approach is, however, empirical as it relies on a 'trial and error' process. Consequently, it can be extremely time-consuming if the number of degrees of freedom is large. A more systematic approach for the open-loop control of vortex-shedding, based on sensitivity analyses, has been introduced by Hill [2] and revisited by Marquet et al. [3]. The main idea underlying these studies is that the effect of a steady control is to modify the base flow on which the perturbation develops. The effect of the control on the flow stability can then be simply estimated from the computation of a gradient (or sensitivity function). These concepts are extended here to the case of nonparallel compressible flows and applied to the control of unsteadiness in a subsonic afterbody flow, for which additional control methods, namely, the addition of heat sources and boundary forcing are considered.

\section{THEORETICAL FRAMEWORK}

Let consider an axisymmetric body of revolution of diameter $D$ and total length $l=9.8 D$, with a blunt trailing edge and an ellipsoidal nose of aspect ratio $3: 1$, placed into a uniform flow at zero angle of attack (Fig. 1). Standard cylindrical coordinates $(r, \theta, z)$ with origin taken at the center of the base are used. The fluid is taken as a nonhomogeneous compressible perfect gas with specific heat $c_{p}$, thermal conductivity $\kappa$, and dynamic viscosity $\mu$, related by a unit Prandtl number. The fluid motion is described by the state vector $\boldsymbol{q}$ $=(\rho, \boldsymbol{u}, T, p)^{\mathrm{T}}$ where $\rho$ is the density; $T$ is the temperature; $p$ is the pressure; and $\boldsymbol{u}=(u, v, w)^{\mathrm{T}}$ is the three-dimensional (3D) velocity field with $u, v$, and $w$ its radial, azimuthal, and streamwise components. The state vector $\boldsymbol{q}$ obeys the unsteady compressible Navier-Stokes equations, thus leading to a set of 6 nonlinear equations (continuity, momentum, internal energy, and perfect gas) formulated in nonconservative variables and made nondimensional using the body diameter and the upstream flow quantities:

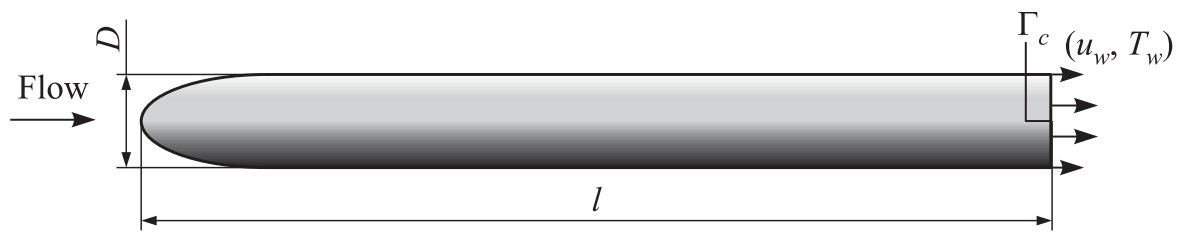

Figure 1 Schematic of the configuration under study: the slender body of revolution has a diameter $D$ and a total length $l=9.8 D$ 


$$
\left.\begin{array}{r}
\partial_{t} \rho+\rho \boldsymbol{\nabla} \cdot \boldsymbol{u}+\boldsymbol{u} \cdot \boldsymbol{\nabla} \rho=m ; \\
\rho \partial_{t} \boldsymbol{u}+\rho \boldsymbol{\nabla} \boldsymbol{u} \cdot \boldsymbol{u}+\frac{1}{\gamma \mathrm{M}^{2}} \nabla p-\frac{1}{\operatorname{Re}} \nabla \cdot \boldsymbol{\tau}(\boldsymbol{u})=\boldsymbol{f} ; \\
\rho \partial_{t} T+\rho \boldsymbol{u} \cdot \nabla T+p \boldsymbol{\nabla} \cdot \boldsymbol{u}-\gamma(\gamma-1) \frac{\mathrm{M}^{2}}{\operatorname{Re}} \boldsymbol{\tau}(\boldsymbol{u}): \boldsymbol{d}(\boldsymbol{u})-\frac{\gamma}{\operatorname{Pr} \operatorname{Re}} \nabla^{2} T=h .
\end{array}\right\}
$$

Here, Re and M are the Reynolds and Mach numbers, whereas $\boldsymbol{d}(\boldsymbol{u})$ and $\boldsymbol{\tau}(\boldsymbol{u})$ are the strain and viscous stress tensors. Equations (1) are formally written as

$$
\mathcal{B}(\boldsymbol{q}) \boldsymbol{q}+\mathcal{M}(\boldsymbol{q}, \mathcal{G})=(\mathscr{S}, 0)^{\mathrm{T}}
$$

where $\mathcal{B}$ and $\mathcal{M}$ are the differential operators and $\mathcal{G}$ represents the set of control parameters (Reynolds and Mach numbers, angle of attack, etc.) which remains constant here, so that the dependence in $\mathcal{G}$ is omitted to ease the notation. The vector $\mathscr{S}=(m, \boldsymbol{f}, h)^{\mathrm{T}}$ defines the bulk forcing, $\boldsymbol{f}$ (in $\mathrm{m} / \mathrm{h}$ ) being the volumetric momentum flux (respectively, volumetric heat and mass fluxes) associated to the control. The effect of wall forcing is also taken into account by adding a subsonic inlet condition at the base, whose surface is denoted $\Gamma_{c}$ :

$$
\boldsymbol{u}=\boldsymbol{u}_{w}, \quad T=T_{w} .
$$

Note that the coefficients $c_{p}, \kappa$ and $\mu$ are assumed to be constant, which may be relevant to the moderate Mach numbers presently investigated. Still, it is worthwhile noting that the theoretical formalism developed hereafter can be generalized to account for the effect of temperature, provided analytical laws are used. As an example, one may compute the viscosity from a standard Sutherland's law, the Reynolds number being then built from the reference viscosity.

In the following, the Mach number is set to $\mathrm{M}=0.5$. The state vector is split into an axisymmetric steady base flow $\boldsymbol{q}^{\mathbf{0}}=\left(\rho^{0}, u^{0}, 0, w^{0}, T^{0}, p^{0}\right)^{\mathrm{T}}$ and a $3 \mathrm{D}$ perturbation $\boldsymbol{q}^{\mathbf{1}}=\left(\rho^{1}, u^{1}, v^{1}, w^{1}, T^{1}, p^{1}\right)^{\mathrm{T}}$ of small amplitude. Here, the case of an axisymmetric steady control only is considered, so that the base flow equations read:

$$
\mathcal{M}_{0}\left(\boldsymbol{q}^{\mathbf{0}}\right)=(\mathscr{S}, 0)^{\mathrm{T}}, \quad \boldsymbol{u}^{\mathbf{0}}=\boldsymbol{u}_{w}, \quad T^{0}=T_{w} \text { on } \Gamma_{c},
$$

where $\mathcal{M}_{0}$ is the axisymmetric form of the evolution operator $\mathcal{M}$. Perturbations are chosen under the form of normal modes:

$$
\boldsymbol{q}^{1}=\hat{\boldsymbol{q}}^{1}(r, z) e^{(\sigma+i \omega) t+i m \theta}+\text { c.c. }
$$

where $\hat{\boldsymbol{q}}_{1}=\left(\hat{\rho}^{1}, \hat{u}^{1}, \hat{v}^{1}, \hat{w}^{1}, \hat{T}^{1}, \hat{p}^{1}\right)^{T}$ is the so-called global mode, characterized by an integer azimuthal wavenumber $m$, growth rate $\sigma$, and pulsation $\omega$. The global mode $\hat{\boldsymbol{q}}^{\mathbf{1}}$ and the complex pulsation $\lambda=\sigma+\mathrm{i} \omega$ are solutions of a generalized eigenvalue problem reading:

$$
\lambda \mathcal{B}\left(\boldsymbol{q}^{\mathbf{0}}\right) \hat{\boldsymbol{q}}^{\mathbf{1}}+\mathcal{A}_{m}\left(\boldsymbol{q}^{\mathbf{0}}\right) \hat{\boldsymbol{q}}^{\mathbf{1}}=\mathbf{0}, \quad \hat{\boldsymbol{u}}^{\mathbf{1}}=\mathbf{0}, \quad \hat{T}^{1}=0 \text { on } \Gamma_{c},
$$


where $\mathcal{A}_{m}$ is the differential operator obtained by linearization of operator $\mathcal{M}$ about $\boldsymbol{q}^{\mathbf{0}}$ and substitution of the $\partial_{\theta}$ terms by the product by $\mathrm{im}$.

A finite elements method is used to discretize Eqs. (2) and (3) on a computational domain $\Omega$ corresponding to the azimuthal plane $\theta=0$, whose boundary is denoted $\Gamma=\Gamma_{w} \cup \Gamma_{\infty}$ where $\Gamma_{w}$ corresponds to the afterbody walls. In order to save computational resources, all pressure terms are eliminated and replaced by their expressions issuing from the perfect gas equations for $p^{0}$ and $\hat{p}^{1}$. Note that either the density or the temperature could have been alternatively eliminated. Nevertheless, the present choice considerably eases the numerical implementation, owing to the limited number of terms to be replaced in the governing equations. The base flow Eqs. (2) are solved using an iterative Newton method [4], and the disturbance Eqs. (3) are solved using a Shift and Invert Arnoldi method [5]). In the present compressible regime, the choice of appropriate far-field radiation conditions may be particularly involved. Consequently, sponge zones are used where all fluctuations are progressively damped to negligible levels through artificial dissipation before they reach the boundary of the computational domain, which requires to use sponge regions whose size is somewhat comparable to that of the inner domain. The boundary conditions satisfied by the base flow and the disturbances are then deduced from that satisfied by the state vector $\boldsymbol{q}=(\rho, \boldsymbol{u}, T)^{\mathrm{T}}$ :

$$
\begin{array}{lll}
\boldsymbol{u}=(0,0,1)^{\mathrm{T}}, & \rho, T=1 & \text { on } \Gamma_{\infty} ; \\
\boldsymbol{u}=\mathbf{0}, & \partial_{n} T=0 & \text { on } \Gamma_{w} \backslash \Gamma_{c} .
\end{array}
$$

Let consider now the unforced flow, for which

$$
m=0, \quad f=\mathbf{0}, \quad h=0, \quad \boldsymbol{u}_{\boldsymbol{w}}=\mathbf{0}, \quad T_{w}=1+\frac{\gamma-1}{2} \mathrm{M}^{2} .
$$

The effect of a small-amplitude forcing on the flow stability is assessed by investigating the variation of a given eigenvalue $\delta \lambda=\delta \sigma+\mathrm{i} \delta \omega$. In the present linear framework, the eigenvalue variation resulting from the introduction of the forcing can be written as the scalar product between the forcing term and a sensitivity function or gradient:

$$
\begin{aligned}
\delta \lambda=\int_{\Omega}\left(\nabla_{m} \lambda \cdot \delta m+\nabla_{\boldsymbol{f}} \lambda \cdot \boldsymbol{\delta} \boldsymbol{f}+\boldsymbol{\nabla}_{h} \lambda \cdot \delta h\right) r d r d z & \\
& +\int_{\Gamma_{c}}\left(\boldsymbol{\nabla}_{\boldsymbol{u}_{\boldsymbol{w}}} \lambda \cdot \boldsymbol{\delta} \boldsymbol{u}_{\boldsymbol{w}}+\boldsymbol{\nabla}_{T_{w}} \lambda \cdot \delta T_{w}\right) r d z
\end{aligned}
$$

where $\cdot$ refers to the canonic hermitian scalar product in $\mathbb{C}^{n}$, and $\nabla_{m} \lambda, \nabla_{f} \lambda$, and $\nabla_{h} \lambda$ are complex vectors defining the sensitivity of the eigenvalue to a source of mass, momentum, and internal energy, respectively. Similarly, $\nabla_{\boldsymbol{u}_{\boldsymbol{w}}} \lambda$ and 
$\nabla_{T_{w}} \lambda$ define the sensitivity of the eigenvalue to a wall velocity and temperature. The analytical expression of these gradients is derived using a Lagrangian method relying on the definition of adjoint variables, an approach similar to that widely used in the context of optimization problems [6]:

$$
\begin{aligned}
& \left(\boldsymbol{\nabla}_{m} \lambda, \boldsymbol{\nabla}_{\boldsymbol{f}} \lambda, \boldsymbol{\nabla}_{h} \lambda\right)^{\mathrm{T}}=\left(\rho^{0 \dagger}, \boldsymbol{u}^{\mathbf{0}}, T^{0 \dagger}\right)^{T} ; \\
& \nabla_{\boldsymbol{u}_{\boldsymbol{w}}} \lambda=\rho^{0} \rho^{0 \dagger} \boldsymbol{n}+\frac{1}{\operatorname{Re}}\left(-\frac{2}{3}\left(\boldsymbol{\nabla} \cdot \boldsymbol{u}^{0 \dagger}\right) \boldsymbol{I}+\boldsymbol{\nabla} \hat{\boldsymbol{u}}^{0 \dagger}+\nabla \hat{\boldsymbol{u}}^{0 \dagger} \mathrm{T}\right) \cdot \boldsymbol{n} ; \\
& \boldsymbol{\nabla}_{T_{w}} \lambda=\frac{\gamma}{\operatorname{Pr} \operatorname{Re}} \nabla T^{0 \dagger} \cdot \boldsymbol{n} .
\end{aligned}
$$

Here, $\boldsymbol{n}$ is the normal to the afterbody wall oriented from the body towards the fluid; and $\boldsymbol{q}^{\mathbf{0}}=\left(\rho^{0 \dagger}, \boldsymbol{u}^{\mathbf{0}}, T^{0 \dagger}\right)^{\mathrm{T}}$ is termed the adjoint base flow and is the solution of the forced linear problem:

$$
\mathcal{A}_{0}^{\dagger}\left(\boldsymbol{q}^{\mathbf{0}}\right) \boldsymbol{q}^{\mathbf{0} \dagger}=-\lambda^{*} \mathcal{R}^{\dagger}\left(\boldsymbol{q}^{\mathbf{0}}, \hat{\boldsymbol{q}}^{\mathbf{1}}\right) \hat{\boldsymbol{q}}^{\mathbf{1} \dagger}-\mathcal{S}_{m}^{\dagger}\left(\boldsymbol{q}^{\mathbf{0}}, \hat{\boldsymbol{q}}^{\mathbf{1}}\right) \hat{\boldsymbol{q}}^{\mathbf{1} \dagger}
$$

where the superscript $*$ designate the complex conjugate. In $(5), \mathcal{A}_{0}^{\dagger}$ is the adjoint of the axisymmetric operator $\mathcal{A}_{0}$, obtained by integrating by parts the axisymmetric, steady form of the disturbance equations (3). Similarly, operators $\mathcal{R}^{\dagger}$ et $\mathcal{S}_{m}^{\dagger}$ are the adjoints of operators $\mathcal{R}$ and $\mathcal{S}_{m}$, defined as

$$
\mathcal{R}\left(\boldsymbol{q}^{\mathbf{0}}, \hat{\boldsymbol{q}}^{\mathbf{1}}\right)=\frac{\partial}{\partial \boldsymbol{q}^{\mathbf{0}}}\left(\mathcal{B}\left(\boldsymbol{q}^{\mathbf{0}}\right) \hat{\boldsymbol{q}}^{\mathbf{1}}\right) ; \quad \mathcal{S}_{m}\left(\boldsymbol{q}^{\mathbf{0}}, \hat{\boldsymbol{q}}^{\mathbf{1}}\right)=\frac{\partial}{\partial \boldsymbol{q}^{\mathbf{0}}}\left(\mathcal{A}_{m}\left(\boldsymbol{q}^{\mathbf{0}}\right) \hat{\boldsymbol{q}}^{\mathbf{1}}\right) .
$$

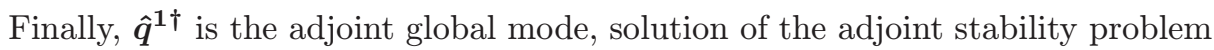

$$
\lambda^{*} \mathcal{B}\left(\boldsymbol{q}^{\mathbf{0}}\right) \hat{\boldsymbol{q}}^{\mathbf{1 \dagger}}+\mathcal{A}_{m}^{\dagger}\left(\boldsymbol{q}^{\mathbf{0}}\right) \hat{\boldsymbol{q}}^{\mathbf{1 \dagger}}=\mathbf{0}
$$

The procedure to be followed to compute the sensitivity of an eigenvalue to a small-amplitude steady forcing acting at the base flow level can be summarized as follows:

(1) resolution of the unforced base flow Eqs. (2);

(2) resolution of the generalized eigenvalue problem (3) and selection of an eigenvalue $\lambda$ and of the associated global mode $\hat{\boldsymbol{q}}^{\mathbf{1}}$;

(3) computation of the adjoint global mode $\hat{\boldsymbol{q}}^{\mathbf{1 \dagger}}$ by resolution of the adjoint stability problem (6);

(4) computation of the right-hand side in (5);

(5) computation of the adjoint base flow by resolution of the linear problem (5); and

(6) computation of the sensitivity functions according to (4). The sensitivity functions relative to the growth rate $\sigma$ are then deduced by retaining only the real parts of these complex sensitivity functions. 


\section{RESULTS}

\subsection{Global Stability Analysis}

The global stability analysis shows that the unforced axisymmetric base flow sustains two subsequent instabilities: the first bifurcation occurs at the critical Reynolds number $\operatorname{Re}_{A}=483.5$ and involves a stationary global mode $A$ of azimuthal wavenumber $m=1$ and frequency $\omega=0$ (not shown here). A Hopf bifurcation then occurs at $\operatorname{Re}_{B}=983$, involving a $m=1$ global mode $B$ oscillating at the frequency $\omega_{B}=0.399\left(\mathrm{St}=f D / U_{\infty}=0.06\right)$. The associated complex eigenvector is from now on referred to as $\hat{\mathbf{q}}_{\mathrm{B}}^{1}$. Mode $B$ exhibits positive and negative velocity perturbations alternating downstream of the body, in a regular, periodic way, as illustrated by the streamwise velocity distribution shown in Fig. 2. These results are in agreement with the global stability analysis carried out by Natarajan and Acrivos [7] in the context of the incompressible flow past disks and spheres, for which mode $B$ dominates the dynamics of the fully $3 \mathrm{D}$ flow at large Reynolds numbers, and triggers the occurrence of a fully $3 \mathrm{D}$ periodic state $[8,9]$. Consequently, in the following, let focus on the effect of open-loop control on the growth rate $\sigma_{B}$ of the oscillating mode $B$.

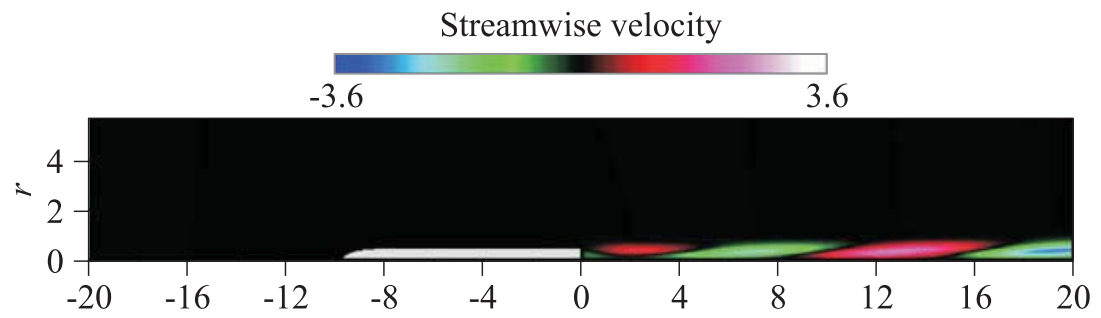

Figure 2 Streamwise velocity component of the oscillating global mode $B$ at the threshold of instability: $\operatorname{Re}_{B}=983.0, \mathrm{M}=0.5$ (the background grey hue stands for vanishing perturbation amplitudes). (Refer Meliga et al., p. 466.)

\subsection{Effect of a Control Ring}

Let study first the effect of a small control device, chosen here as a ring of width $e$ and radius $r_{c}$, mounted at the rear of the main body, at a distance $z_{c}$ of the base. As in the studies of Hill [2] and Marquet et al. [3], it is considered that the flow exerts a localized drag force on the control ring and that the ring exerts in return an opposite force on the flow, modeled as

$$
\boldsymbol{\delta} \boldsymbol{f}(r, z)=-\frac{1}{2} C e \rho^{0}(r, z)\left\|\boldsymbol{u}^{\mathbf{0}}(r, z)\right\| \boldsymbol{u}^{\mathbf{0}}(r, z) \delta\left(r-r_{c}, z-z_{c}\right)
$$




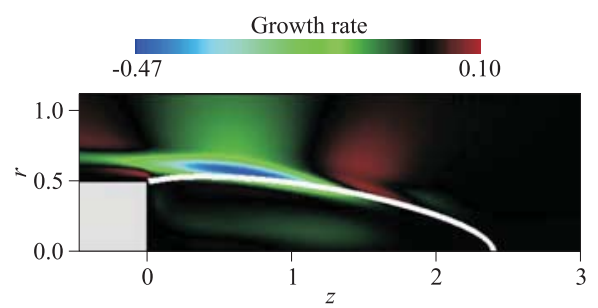

(a)

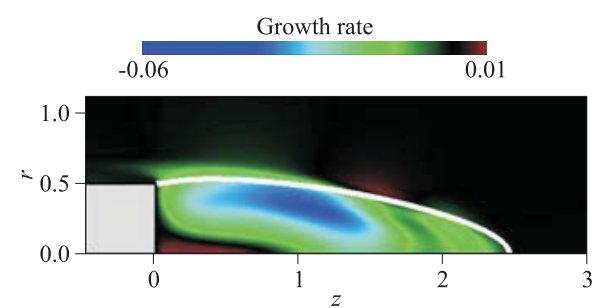

(b)

Figure 3 Spatial distribution of the growth rate variation $\delta \sigma_{B}\left(r_{c}, z_{c}\right)$ obtained when the presence of a small control ring is modeled by the force $(7)(a)$ and when using a heat source modeled by (16) with $\delta \hat{h}=10^{-2}(b)$ : $\mathrm{Re}=983, \mathrm{M}=0.5$. The background grey hue stands for vanishing variations. (Refer Meliga et al., p. 467.)

where $C$ is the drag coefficient depending on the value of the Reynolds number $\operatorname{Re}_{e}$ built from the ring width and the local flow velocity. Typical values of $\operatorname{Re}_{e}$ in the recirculating bubble are of order $\operatorname{Re}_{e} \simeq 30$, so that it is chosen here $C=1$, an empirical value estimated from the drag coefficient of a circular cylinder in this range of Reynolds numbers. To each position of the ring $\left(r_{c}, z_{c}\right)$ corresponds a variation of the growth rate $\delta \sigma_{B}$ that can be expressed simply as the scalar product between the induced force $\delta \boldsymbol{f}$ and the sensitivity function $\boldsymbol{\nabla}_{\boldsymbol{f}} \sigma_{B}$, i. e.,

$$
\delta \sigma_{B}^{0}\left(r_{c}, z_{c}\right)=-\frac{1}{2} \operatorname{Cer}_{c} \rho^{0}\left(r_{c}, z_{c}\right)\left\|\boldsymbol{u}^{\mathbf{0}}\left(r_{c}, z_{c}\right)\right\| \nabla_{\boldsymbol{f}} \sigma_{B}\left(r_{c}, z_{c}\right) \cdot \boldsymbol{u}^{\mathbf{0}}\left(r_{c}, z_{c}\right) .
$$

Figure $3 a$ presents the spatial distribution of the growth rate variation $\delta \sigma_{B}\left(r_{c}, z_{c}\right)$. Since the global mode is marginally stable, negative variations $\delta \sigma_{B}$ $<0$ (respectively, positive variations $\delta \sigma_{B}>0$ ) correspond to a stabilization (respectively, a destabilization) of the global mode. It was found that the ring induces a strong stabilization if placed along the separation line. However, it should be noted that the effect of such momentum forcing is complex, since several regions contributing either to a weak stabilization or destabilization of the global mode are visible around the main stabilizing region.

\subsection{Effect of a Heat Source}

Let consider now the effect of a localized heat source modeled by

$$
\delta h(r, z)=\frac{1}{2 \pi r_{c}} \delta \hat{h} \delta\left(r-r_{c}, z-z_{c}\right)
$$


Physically, $\delta \hat{h}$ is the flux of internal energy imposed by the control, so that a positive (respectively, negative) value of $\delta \hat{h}$ corresponds to a heating (respectively, a cooling) of the flow. Again, to each position of the source corresponds a growth rate variation $\delta \sigma_{B}$ given by the scalar product between the forcing term $\delta h$ and the sensitivity function $\nabla_{h} \sigma_{B}$ :

$$
\delta \sigma_{B}^{0}\left(r_{c}, z_{c}\right)=\frac{1}{2 \pi} \nabla_{h} \sigma_{B}\left(r_{c}, z_{c}\right) \delta \hat{h} .
$$

In Fig. $3 b$, the results obtained for $\delta \hat{h}=10^{-2}$ are shown, i. e., the flow is heated and the cost of the control represents $1 \%$ of the internal energy flux of the incoming flow. It was found that heating the flow within the recirculating area has a stabilizing effect, whatever the position of the source. The maximum stabilizing effect obtained by this method corresponds to a variation $\delta \sigma_{B}=-0.06$ and is, however, less important than that achieved using the control ring.

\subsection{Effect of Wall Blowing-Base Bleed}

Let consider now the case of a wall forcing where fluid is blown through the base, the wall temperature being unchanged. Only the case of a wall-normal blowing velocity $\delta \boldsymbol{u}_{w}=\delta w_{w} \boldsymbol{e}_{\boldsymbol{z}}$ will be investigated, so that the growth rate variation $\delta \sigma_{B}$ reads:

$$
\delta \sigma_{B}=\rho^{0} \rho^{0 \dagger} \delta w_{w}+\frac{1}{\operatorname{Re}}\left(-\frac{2}{3} \nabla \cdot \boldsymbol{u}^{0 \dagger}+2 \partial_{z} w^{0 \dagger}\right) \delta w_{w} .
$$

The variation $\delta \sigma_{B}$ arise from two distinct contributions: the product $\rho^{0} \rho^{0 \dagger}$ corresponds to the effect of the additional mass flux, whereas the contribution weighted by the inverse of the Reynolds number corresponds to the modification of the viscous forces applied at the base. The solid line in Fig. 4 shows the distribution of the sensitivity function $\nabla_{w_{w}} \sigma_{B}$ as a function of the radial position $r$ at the base, at the threshold of the instability $(\operatorname{Re}=983, \mathrm{M}=0.5)$. The sensitivity is negative whatever the position at the base, meaning that blowing fluid $\left(\delta w_{w}>0\right)$ has systematically a stabilizing effect, a result consistent with the documented effect of such strategies. The level of sensitivity is almost constant at the center of the base $(r<0.3)$, but it increases dramatically as one moves closer to the edge, the maximum value being reached in the vicinity of the separation point. Therefore, an actuator imposing a steady blowing will achieve maximum efficiency if placed at the edge of the base. The dashed line in Fig. 4 shows the same sensitivity function, now computed at the supercritical Reynolds number $\operatorname{Re}=2000$, for which the unforced growth rate is $\sigma_{B}=8.5 \cdot 10^{-2}$. Interestingly, it was found that the sensitivity level has significantly increased with 


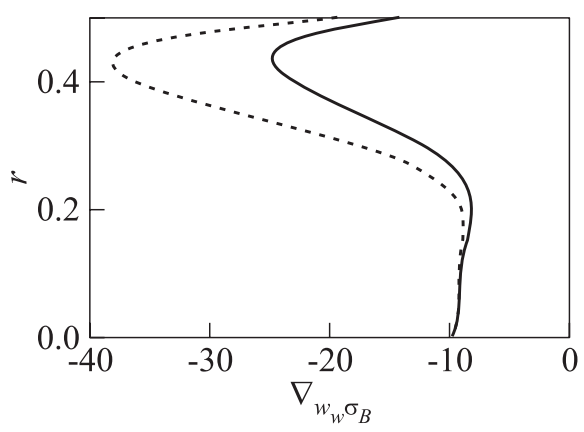

Figure 4 Spatial distribution of the sensitivity function $\nabla_{w_{w}} \sigma_{B}$ to a blowing velocity as a function of the radial position at the base, for $\mathrm{Re}=983$ (solid line) and $\mathrm{Re}=2000$ (dashed line); $\mathrm{M}=0.5$

the Reynolds number, which opens the way to the control of unstable configurations. Using the sensitivity function defined in (9), we obtain a complete stabilization of the global mode $B$ for a uniform blowing velocity $\delta w_{w} \simeq 0.03$. However, it is possible to use the sensitivity function to optimize the blowing velocity, by increasing (respectively, reducing) the blowing velocity in the base region where the sensitivity level is high (respectively, low), the blowing flow rate being kept constant. This makes it possible to achieve a complete stabilization with a critical flow rate smaller by $8 \%$ than that found in the uniform case (not shown here).

\section{CONCLUDING REMARKS AND PERSPECTIVES}

This study presents sensitivity analyses to a steady forcing, applied to a subsonic afterbody flow. These analyses allow to determine which open-loop control strategies are most efficient to alleviate the unsteadiness arising in this class of flows. It was shown that the global mode responsible for the onset of unsteadiness can be stabilized by placing a small control ring close to the separation line, by heating the flow within the recirculating area or by blowing fluid through the base.

Currently, the authors aim at using this formalism to interpret physically the stabilizing effects documented here. This is done by carrying out similar sensitivity analyses, where the growth rate variation is no more investigated as a function of the forcing, but as a function of the base flow modification induced by the forcing. The results obtained so far suggest that all control strategies investigated act in the same way, namely, they modify the base flow momentum distribution which results in an increase of the disturbances advection. 


\section{REFERENCES}

1. Strykowski, P. J., and K. R. Sreenivasan. 1990. On the formation and suppression of vortex shedding at 'low' Reynolds numbers. J. Fluid Mech. 218:71-107.

2. Hill, D. C. 1992. A theoretical approach for analyzing the restabilization of wakes. Technical Report 103858. NASA.

3. Marquet, O., D. Sipp, and L. Jacquin. 2008. Sensitivity analysis and passive control of the cylinder flow. J. Fluid Mech. 615:221-52.

4. Barkley, D., M. G. M. Gomes, and R. D. Henderson. 2002. Three-dimensional instability in flow over a backward-facing step. J. Fluid Mech. 473:167-90.

5. Ehrenstein, U., and F. Gallaire. 2005. On two-dimensional temporal modes in spatially evolving open flows: The flat-plate boundary layer. J. Fluid Mech. 536:209-18.

6. Gunzburger, M. D. 1999. Sensitives, adjoints and flow optimization. Int. J. Numer. Meth. Fluids 31(1):53-78.

7. Natarajan, R., and A. Acrivos. 1993. The instability of the steady flow past spheres and disks. J. Fluid Mech. 254:323-44.

8. Fabre, D., F. Auguste, and J. Magnaudet. 2008. Bifurcations and symmetry breaking in the wake of axisymmetric bodies. Phys. Fluids 20(5):051702 1-4.

9. Meliga, Ph., J.-M. Chomaz, and D. Sipp. 2009. Global mode interaction and pattern selection in the wake of a disk: A weakly nonlinear expansion. J. Fluid Mech. 633:159-89. 